\title{
Detailed imaging of mitochondrial transport and precise manipulation of mitochondrial function with genetically-encoded photosensitizers in adult Drosophila neurons
}

\section{Francesca Mattedi}

King's College London https://orcid.org/0000-0002-0306-5796

George Chennell

King's College London https://orcid.org/0000-0001-5859-361X

Alessio Vagnoni ( $\square$ alessio.vagnoni@kcl.ac.uk)

King's College London https://orcid.org/0000-0003-2947-9193

\section{Method Article}

Keywords: Drosophila, mitochondria, reactive oxygen species (ROS), KillerRed, SuperNova, axonal transport, neurons, intravital imaging, super-resolution radial fluctuations (SRRF)

Posted Date: May 21st, 2021

DOl: https://doi.org/10.21203/rs.3.rs-540726/v1

License: (c) (1) This work is licensed under a Creative Commons Attribution 4.0 International License.

Read Full License 


\section{Abstract}

Precise distribution of mitochondria is essential for maintaining neuronal homeostasis. Although detailed mechanisms governing the transport of mitochondria have emerged, it is still poorly understood how the regulation of transport is coordinated in space and time within the physiological context of an organism. How alteration in mitochondrial functionality may trigger changes in organellar dynamics also remains unclear in this context. Therefore, the use of genetically-encoded tools to perturb mitochondrial functionality in real time would be desirable. Here we describe methods to interfere with mitochondrial function with high spatiotemporal precision with the use of photosensitisers in vivo in the intact wing nerve of adult Drosophila. We also provide details on how to visualise the transport of mitochondria and to improve the quality of the imaging to attain super-resolution in this tissue.

\section{Introduction}

Mitochondria are highly dynamic organelles and their correct distribution is crucial to support many cellular functions. The remarkably long processes of neurons mean that these cells are particularly dependent on mechanisms for long-range cytoskeletal transport of mitochondria. Due to their peculiar architecture, and the stereotypical directionality of the transport in the axons, neurons are a particularly good model to understand the logic of intracellular trafficking.

The fundamental importance of axonal transport for achieving maturation and maintaining the functionality of neurons is well documented [1] and many models have been developed to visualise this process. High throughput, ease of gene manipulation and accessibility to pharmacological treatments make cultured neuronal cells, for example primary neurons or neurons derived from neuroblastoma and stem cells, arguably the mainstay of neuronal trafficking studies and have significantly contributed towards the mechanistic understanding of the transport process [2-13]. Ex vivo models, where a whole tissue is dissected and maintained in culture or imaged directly after dissection, are valid alternatives [14-20] with the advantage of potentially preserving a near-native environment while affording accessibility for pharmacological and electrophysiological studies.

In vivo animal models to study the transport process in neurons are now also widely used [6, 21-29]. A clear advantage of an in vivo system is the possibility of studying the transport process while retaining the full complexity of an organismal setting, which may be preferable when studying axonal transport during ageing and in animal models of age-dependent neurodegenerative disorders [30]. However, because the neurons of interest are often buried deep into the tissue that is being imaged, this advantage tends to be offset by the need of complex surgery required to visualize the neurons of interests. This is the case, for example, for the implantation of cranial windows for the observation of thalamo-cortical projections in mice [31] or for the exposure of the mouse sciatic nerve for trafficking studies [32]. In addition, the resolution at which dynamic subcellular components are resolved during in vivo imaging is often modest compared to the quality that can be obtained from in vitro cultured cells. Despite the current rapid development of super-resolution techniques, only few in vitro studies have focused on long-range 
intracellular transport captured by live-cell super-resolution imaging $[33,34]$. While challenging, achieving super-resolution of trafficked organelles in vivo would be desirable, and a tangible step forward, to better understand the intricacies of intracellular trafficking in this context.

Performing gene manipulations to interfere with the transport or function of specific cargoes might not be straightforward in vivo, especially in mammalian systems. Knockdown or knockout studies are timeconsuming; acute modulation of transport, for instance by the addition of drugs, also presents challenges in an in vivo setting, limiting the possibility of gaining in-depth mechanistic insight of this process. This is problematic when studying mitochondrial dynamics which often relies on pharmacological manipulation to interfere with different stages of the oxidative phosphorylation.

We developed a system for detailed imaging of organelle transport in adult Drosophila in vivo, which exploits the accessibility of the wing neurons for microscopic observation and does not require surgical procedures [35]. In this chapter, we describe methods to image mitochondrial transport in different neuronal population of the wing using anaesthetized adult animals. We have previously showed the suitability of this tissue for structured illumination microscopy (SIM) of neuronal membranes and nuclear markers [35]. With the aim of improving the resolution for in vivo imaging of mitochondrial trafficking, here we describe the application of Super-Resolution Radial Fluctuation (SRRF) microscopy [36, 37] to live imaging in the wing nerve of adult Drosophila. Finally, we report the generation of new transgenic flies encoding the photosensitisers KillerRed and SuperNova targeted to the mitochondria and describe photostimulation protocols to induce mitochondrial damage rapidly, coupled to the imaging of the redox state and motility of the organelles. We believe this will expand the utility of the wing nerve system and provide additional tools for the study of mitochondrial function and transport in vivo.

\section{Materials}

\subsection{Generation of plasmids for the production of transgenic fly lines}

1. Plasmids containing the mitochondrial targeting sequence (MTS) of the human cytochrome $c$ oxidase subunit VIII (Cox VIII), KillerRed and SuperNova coding sequences. We used an 2x-MTSmCherry (Vagnoni lab collection), \#45761 and \#53234 from Addgene for KillerRed and SuperNova, respectively.

2. PCR primers (underlined, sequences complementary to the coding sequences of interest; italic, overlapping sequences - see also section 3.1):

A) 2x-MTS Forward (FWD): 5'-GCGTTAGGTCCTGTTCATTGgt

acCATGTCCGTCCTGACGCCGCTGCTGCTGCGG-3'; Reverse (RVS) with overlapping sequence to KillerRed: 5'-AGGGCGGGGCCGCCCTCGGAggatc cCAACGAATGGATCTTGGCGCGCGGCACTGG-3'; RVS with overlapping sequence to SuperNova: 5'-GGGCCGACCTCTGAACCCATggatccCAACGA ATGGATCTTGGCGCGCGGCACTGG-3' 
B) KillerRed

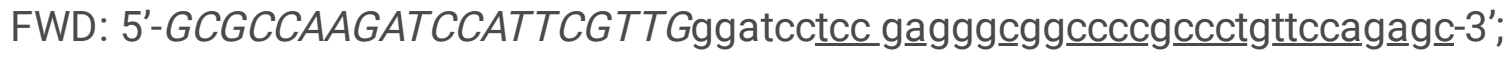

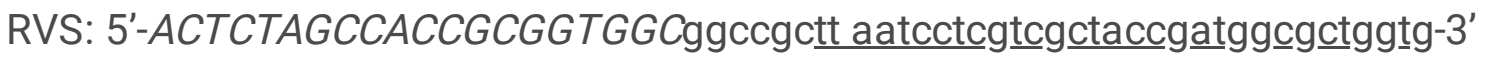

C) SuperNova

FWD: 5'-GCGCCAAGATCCATTCGTTGggatccatg ggttcagaggtcggccccgccctgttc-3'; RVS: 5'ACTCTAGCCACCGCGGTGGCggccgcttaatc ctcgtcgctaccgatggcgctggtg-3'.

3. pUASP plasmid for P-element mediated transgenesis.

\subsection{Fly maintenance}

1. Fly food: $70 \mathrm{mg} / \mathrm{ml}$ yeast, $55 \mathrm{mg} / \mathrm{ml}$ glucose, $7.7 \mathrm{mg} / \mathrm{ml}$ agar, $35 \mathrm{mg} / \mathrm{ml}$ organic plain white flour, 1.2 $\mathrm{mg} / \mathrm{ml}$ Tegosept, $0.4 \%$ propionic acid (see Note 1 ).

2. Adult flies: adult transgenic animals expressing a fluorescent reporter to mark the mitochondria in the wing marginal nerve. The stocks used in this study are described in Table 1.

3. Incubator equipped with temperature and humidity control, and 12-h-light/12-h-dark cycle (see Note 2).

\subsection{Sample preparation for imaging}

1. Coverslips no. 0,1 or 1.5 (see Note 3).

2. $10 \mathrm{~S}$ halocarbon oil (see Note 4).

3. Dumont forceps \#5.

4. Round tip artist paintbrush for Drosophila

5. Masking tape and double-sided tape.

6. Stereo microscope for Drosophila

\subsection{Imaging axonal transport in the Drosophila wing and photostimulation}

1. Inverted confocal microscope equipped with $40 x, 60 x$ or $100 x$ oil immersion objective and with temperature control. For photostimulation experiments, the microscope should be equipped with a photostimulation module (see Note 5).

2. ImageJ or Fiji equipped with RatioProfiler plugin (https://imagej.net/Cookbook) and the 'Velocity measurement tool' (http://dev.mri.cnrs.fr/projects/imagej-macros/wiki/Velocity_Measurement_Tool) 
for kymographs generation (see Note 6).

3. NIS-element software (Nikon) for image processing.

4. Microsoft Excel and GraphPad Prism to analyse and plot the data.

\subsection{SRRF analysis}

ImageJ or Fiji equipped with NanoJ Core and NanoJ-SRRF plugins [38].

\section{Methods}

We use the GAL4-UAS binary system [39] to drive the expression of GFP in the mitochondria of the adult wing neurons. By using either a pan-neuronal driver, for example $n S y b-G a / 4$, or a more restricted driver such as the cholinergic neuronal driver ChAT-Gal4, mitochondria can be visualised clearly in the neurons of the adult Drosophila wing (Figure 1B-D) (see Note 7). However, application of the SRRF algorithm to time series acquired by spinning disk microscopy allows to achieve a considerably improved resolution of both stationary and moving mitochondria (Figure 1D and Movie 1), which is useful to appreciate finer details of mitochondrial dynamics.

In order to damage mitochondria with spatiotemporal precision, we produced novel transgenic Drosophila lines expressing the photosensitisers KillerRed (KR) [40] and SuperNova (SN) [41] targeted to the mitochondrial matrix (mito::KR and mito::SN, respectively) (Table 1, Figure 2 and Figure 5A). Upon irradiation with green light, KR and SN produce a large amount of reactive oxygen species (ROS). As direct readout of the efficacy of our stimulation protocol, we measured the mitochondrial redox state with the roGFP2-Grx1 reporter (mito::roGFP2-Grx1), which exhibits fluorescence emission shift from $488 \mathrm{~nm}$ to $405 \mathrm{~nm}$ in more oxidised mitochondrial environments [42, 43]. Brief stimulations of mito::KR with a 561$\mathrm{nm}$ laser light are sufficient to trigger a rapid and transient increase in mitochondrial oxidation (Figure 3 ). Our protocol allows to stimulate areas covering a large portion of the nerve (Figure 3 ) as well as achieving precise photostimulation of a single mitochondrion (Figure 4). Mito::KR ${ }^{-}$neurons that do not express mito::KR do not show a detectable mitochondrial oxidative response when subjected to the same photostimulation regime, ruling out the possibility that the exposure to the 561-nm laser light alone is sufficient to strongly increase mitochondrial oxidation, at least for the brief irradiation times used here. No significant change in the mitochondrial redox state was observed after brief stimulation of mito::SN with a 561-nm laser light (Figure 5B-D). This suggests heightened sensitivity to mito::KR in our system which we find advantageous to be able to probe the early consequences of mitochondrial oxidation while minimising light-induced phototoxicity. SuperNova is a monomeric variant of KillerRed and was shown to generate a different proportion of singlet oxygen and superoxide species compared to KillerRed [41, 44]. This was not reported to diminish its phototoxic effect [41], although a robust effect of SuperNova stimulation might become clearer on longer timescales in vivo or after longer irradiation times [45]. SuperNova2, a variant of SuperNova with enhanced phototoxicity has been recently engineered [46] and could be a valid alternative to enhance the phototoxicity of the protein in an in vivo setting. 
To investigate the consequences of acute mitochondrial damage on the motility of the organelle, we present two specular approaches. In the first approach, KillerRed is bleached in mito::KR mito::GFP neurons and the damaged mitochondria undergo a yellow-to-green colour switch (Figure 6A and Movie 2). An alternative approach is to use mito::KR in combination with a mitochondrially-targeted photoactivatable GFP (mito::PA-GFP). In this case, simultaneous stimulation with 405-nm and 561-nm lasers leads to quenching of the red signal and concomitant activation of the green fluorophore, with mitochondria undergoing a red to green transition (Figure 7A and Movie 3). Co-expression of the two mitochondrial markers does not affect the motility and morphology of the organelles and, with both approaches, irradiated (i.e., dysfunctional) mitochondria can be tracked through colour switch. However, the use of PA-GFP is preferable when the aim is to study the dynamics of the irradiated mitochondria, as only one laser line $(488 \mathrm{~nm})$ is required to directly follow the mitochondria after photostimulation. To study the dynamics of both irradiated and non-irradiated mitochondria, the simultaneous use of both 488-nm and 561-nm laser lines for the whole duration of image acquisition is recommended.

\subsection{Generation of plasmids for the production of transgenic lines}

1. The Cox VIII MTS, KillerRed and SuperNova are PCR amplified using the Q5® Hot-Start Fidelity $2 X$ Master Mix (NEB) and FWD and RVS primers reported in section 2.1, which include overlapping sequences complementary to adjacent fragments and necessary for the assembly as described in step 3.

2. The PCR products are purified through solid-phase purification using spin columns (we routinely use Monarch ${ }^{\circledR}$ PCR\&DNA Cleanup kit, NEB).

3. The NEBuilder® HiFi assembly method is used to introduce the KillerRed and SuperNova in the pUASP vector, downstream of two MTS from the human cytochrome coxidase subunit VIII (see Note 8).

\subsection{Fly maintenance}

1. Cross males and virgin females to obtain flies of the desired genotype. In our protocol, the Gal4 drivers and UAS responders are heterozygous in all genotypes.

2. Keep the crosses at $25^{\circ} \mathrm{C}$ with a $12 \mathrm{~h}$-light/12h-dark cycle.

3. Remove the flies after 4-5 days and keep the vial with wandering larvae.

4. Collect newly emerged flies and select the desired genotype. Put the selected flies in new vials, maintain them at $25^{\circ} \mathrm{C}$ with a $12 \mathrm{~h}$-light/12h-dark cycle and image them after $24-48 \mathrm{~h}$, when approximately $30 \%$ of mitochondria are motile [47]. 


\subsection{Sample preparation}

1. Anesthetise the flies on a $\mathrm{CO}_{2}$ pad (see Note 9), while preparing the imaging chamber (Figure $1 \mathrm{~A}$ ).

2. On each of the short sides of the coverslip, make a stack of three layers of masking tape.

3. Add a piece of double-sided tape in the middle of the coverslip and on each stack of masking tape.

4. Apply a thin layer of $10 \mathrm{~S}$ halocarbon oil on one side of the double-sided tape in the middle of the coverslip.

5. Mount one fly on the coverslip. Pick the fly up by the legs and place it on the oil, with the ventral side up and with the head on the double-sided tape in the centre of the coverslip.

6. Dip a fine paintbrush in oil and use it to spread out the wings. Make sure that the wings are flat on the coverslip and most air bubbles are removed (see Note 10).

7. Place a second coverslip on top to seal the chamber and image the fly immediately. To minimise delay, we recommend mounting the fly in proximity of the microscope used for live imaging.

\subsection{Imaging axonal transport in the Drosophila wing}

1. Set the microscope temperature controller at $25^{\circ}$

2. Mount the flies expressing a fluorescence marker (for instance, GFP) targeted to neuronal mitochondria.

3. Find the region of interest in the Drosophila wing by using the transmitted light (through eyepieces).

4. Inspect the whole wing for damage (for instance, small cuts). If the wing is damaged, discard the chamber and mount another fly.

5. Switch to fluorescence mode to check the fluorescent signal (through eyepieces) and then to confocal mode (through microscope software) to acquire the images.

6. Adjust exposure time and laser power to obtain a good signal, clearly visible above background. Here, we used exposure time $300-500 \mathrm{~ms}$ and $15-25 \%$ laser power.

7. Image mitochondrial transport at $1 \mathrm{fps}$ (Figure 1C) (see Note 11).

\subsection{SRRF imaging}

1. Repeat steps 1-5 reported in section 0 .

2. Adjust exposure time and laser power to obtain a good signal, clearly visible above background. Here, we used 30 ms exposure time and 50-60\% laser power (see Note 12).

3. Change the size of the field of view so that the time to acquire a single frame is shorter than the chosen exposure time (see Note 13).

4. Acquire a series of 33 frames by continuous imaging, i.e., where the interval between each frame is limited by the exposure time, in this case $30 \mathrm{~ms}$. Each series of 33 frames will be used to create a 
single SRRF timepoint (see section 3.6).

5. Repeat the 33-frame acquisition with an interval of 1 or $2 \mathrm{~s}$, depending on the desired time lapse between acquisitions ( $0.5 \mathrm{fps}$ and $0.33 \mathrm{fps}$, respectively) (see Note 14).

\subsection{SRRF analysis}

1. Open the raw data in Fiji having installed the Nano-SRRF plugin (Update > Manage Update Sites: add NanoJ-Core and NanoJ-SRRF and complete update process).

2. Calculate the dataset drift using the NanoJ-Core Drift estimation tool in Fiji which computes the sample drift and yields a table later applied to SRRF processing (Plugins $>$ NanoJ-Core $>$ Drift Correction > Estimate Drift). Set the time averaging for drift estimation to the number of frames taken per SRRF time point (in this case 33 frames) with the reference frame set to 'previous frame'.

3. Configure SRRF Temporal Analysis setting to Temporal Radiality Pairwise Product Mean (TRPPM) (Plugins > NanoJ-SRRF > SRRF Configure Advanced Settings). Here, the SRRF analysis settings were set as follows: Ring Radius $=0.3$ and a Radiality Magnification $=2$ (see Note 15).

4. For easier presentation, the results can be imported to NIS-Elements and converted from 32-bit float to 16-bit images. With our settings, the final result is an image sequence of 30 SRRF frames ('SRRF' in Movie 1).

5. To create a movie of the original data with the same frame number to the one obtained through SRRF analysis, generate the mean of 33 frames for each acquisition series, which corresponds to a single SRRF time point. With our settings, this results in a final image sequence composed of 30 frames ('Original' in Movie 1).

\subsection{Photostimulation in the Drosophila wing: monitoring mitochondrial redox state upon acute mitochondrial damage}

1. Repeat steps 1-5 of section 3.4. In this case, select mito::KR mito::roGFP2-Grx1 or mito::SN mito::roGFP2-Grx1 Flies expressing only mito::roGFP2-Grx1 can be used to control for potential lightdependent, photosensitiser-independent, mitochondrial damage.

2. Set up two different optical configurations to image simultaneously roGFP2-Grx1 (A) and the photosensitisers (B):

(A) create two channels with different excitation lasers (405-nm laser and 488-nm laser) and same emission filter set (525/50 bandpass filter).

(B) Use the 561-nm excitation laser and collect the emission signal with a 595/50 bandpass filter.

3. Define the stimulation area by drawing a stimulation ROI (Figure 3A-B) and save it (see Note 16). 
4. Set up an imaging protocol with three steps: 'before stimulation', 'stimulation', 'after stimulation'.

5. Before stimulation: acquire 2-5 frames using optical configurations (A) and (B) from step 2.

6. Stimulation: to efficiently and precisely bleach mito::KR and mito::SN, use a $561-\mathrm{nm}$ laser with $95 \%$ $(3.98 \mathrm{~mW})$ laser power, $16 \mathrm{fps}$ scan speed and 31 loops of stimulation for the cell bodies and axons (ROI area: $65 \mu \mathrm{m}^{2}$ ) or 15 loops for single mitochondria (ROI area: $2.9 \mu \mathrm{m}^{2}$ ). With this configuration, the total duration of the stimulation step never exceeds $3 \mathrm{~s}$ (see Notes 17 and 18).

7. After stimulation: acquire 1 frame every $30 \mathrm{~s}$ for 5 min using optical configurations (A) and (B) from step 2.

\subsection{Photostimulation in the Drosophila wing: assessing mitochondria motility upon acute mitochondrial damage}

1. Repeat steps 1-5 reported in paragraph 3.4. In this case, select mito::KR mito::PA-GFP or mito::KR mito::GFP

2. Define the stimulation area by drawing a stimulation $\mathrm{ROI}$ and save it (see Note 19).

3. Set up an imaging protocol with three phases: 'before stimulation', 'stimulation', 'after stimulation'.

4. Before stimulation: acquire 1 frame every $2.5 \mathrm{~s}$ for $15-20 \mathrm{~s}$.

5. Stimulation: set a scanning speed of $16 \mathrm{fps}$. Use $561-\mathrm{nm}$ laser with $95 \%(3.98 \mathrm{~mW})$ laser power and 62 loops to bleach mito::KR (ROI area: $141 \mu \mathrm{m}^{2}$ ). For simultaneous mito::KR bleaching and mito::PAGFP activation, use 561-nm laser with $80 \%$ (3.39 mW) power and 405-nm laser with 100\% (0.388 $\mathrm{mW}$ ) power and scan simultaneously with 79 loops (ROI area: $90 \mu \mathrm{m}^{2}$ ). With these configurations, the total amount of time for the stimulation step never exceeded $8 \mathrm{~s}$.

6. After stimulation: acquire a frame every $2.5 \mathrm{~s}$ for $2 \mathrm{~min}$.

\subsection{Analysis of mitochondrial redox state upon acute mitochondrial damage}

1. Open the ".nd2" file in Fiji and split the channels.

2. Select the mito::roGFP2-Grx1 channels (Channel 1 and Channel 2) and run "Subtract background" (Process $>$ Subtract background). Define the rolling radius according to the pixel size (in this case, it was set to $20 \mathrm{px}$ ). Channel 1 and Channel 2 represent the fluorescent signal after excitation with the 405-nm and the 488-nm laser, respectively.

3. Merge Channel 1 and 2, in this order, and save the file as ".tiff".

4. Open the stimulation ROI used to bleach the mito::KR signal (Section 3.7, step 3) in the ROI manager.

5. Overlay the stimulation ROI on the ".tiff" file from step n.3. 
6. Run "Ratio profiler" (Cookbook > Image Intensity Processing > Ratio Profiler). A "Results" tab will open and report the ratio between Channel 1 and Channel 2 for each frame. This value is the $405 \mathrm{~nm}$ / $488 \mathrm{~nm}$ ratio for the mito:::roGFP2-Grx1 probe, used to quantify the mitochondrial redox state.

7. Produce ratio images for the visualisation of mitochondrial redox changes (Figure 3A-B, 4A-B, 5B) (see Note 20).

8. To control for the specificity of the irradiation to the region defined by the ROI, run "Ratio profiler" again on an area that has not been stimulated (Figure 3C-D, 5C-D)(see Note 21).

9. In order to obtain the fold change of mitochondrial redox state over time, normalise the $405 \mathrm{~nm} / 488$ $\mathrm{nm}$ ratio at each time point to the mean $405 \mathrm{~nm} / 488 \mathrm{~nm}$ prior to stimulation (Figure 3C-D, 4C, 5C-D).

\subsection{Analysis of mitochondria motility upon acute mitochondrial damage}

1. Open the ".nd2" file in Fiji and split the channels.

2. Run "Subtract background" on each channel and apply a filter to reduce the noise, if needed. For instance, use "Despeckle" (Process > Noise > Despeckle) or "Smooth" (Process > Smooth) in Fiji.

3. Crop the image to analyse only the area of interest. Here we consider a nerve portion of $70 \mu \mathrm{m}$ in length (see Note 22).

4. Open the "Velocity measurement tool" (use the >> button on the Fiji/ImageJ launcher to select this toolset).

5. Draw a straight line along the axons of interest. Define the thickness of the line to include all the axons of interest for kymograph generation. Here, we used 20-px-thick line, running along the whole length of the selected portion of the nerve (Figure 6B and 7B).

6. Press the " $k$ " button to generate the kymograph for each channel.

7. Merge the two kymographs (Figure $6 \mathrm{C}$ and $7 \mathrm{C}$ ).

\section{Notes}

1. Flies were grown on 'Iberian' food, although other recipes are also suitable for visualisation of cargo transport in wing neurons. It is crucial, however, to be consistent with the recipe used as changes in the diet can influence the outcome of the experiments. We found that inconsistent use of the yeast and flour can be the source of experimental variability.

2. To minimise variability in the experimental outcome, it is important that temperature, humidity and light-dark cycle are maintained constant.

3. The shorter working distance afforded by the coverslips n. 0 means that acquiring clear images is straightforward. However, these coverslips are thin and very flexible, which makes stabilising the sample in the imaging chamber more difficult and might lead to drifting while imaging. We find coverslips $\mathrm{n}$. 1 to be a good compromise between quality of images obtained, sample stability and 
maintenance of an appropriate optical configuration. However, coverslips n.1.5 are recommended for live super-resolution imaging (e.g., instant SIM).

4. We compared the $10 \mathrm{~S}$ halocarbon oil from both VWR (cat. no. 24627.188) and the halocarbon oil 700 from Sigma Aldrich (cat. no H8898). We found the former to be more viscous and to better aid sample stabilisation. However, using the Sigma oil does not prevent obtaining high-quality images.

5. Different microscopes can be used to this purpose. We find spinning disk confocal to be the best option for live imaging, as it is gentler on the sample (i.e., less phototoxicity) and affords both highresolution and high-speed. We routinely use two types of confocal settings: a Nikon Ti-E inverted system equipped with a Yokogawa-CSU-X1 Spinning Disk head, Andor iXon Ultra DU-897 EMCCD Camera (for axonal transport studies and SRRF) and an inverted Nikon A1RHD confocal microscope equipped with both GaAsP, multi-alkali and spectral detectors equipped with a photostimulation unit (for light-induced activation of the photosensitisers). With both systems, imaging was performed with a Nikon 60X/1.4NA Plan Apochromatic oil immersion objective.

6. Other kymograph analysis bundles can also be used effectively in this system, for example, KymoClear and KymoDirect [48, 49], KymoAnalyzer [50] or KymoButler [51]. Alternatively, a kymograph of only motile mitochondria could be generated from manually annotated tracks. This would eliminate the background associated with the numerous stationary mitochondria in the wing neurons.

7. The ChAT-Gal4 driver can be used effectively to visualise mitochondria in the wing neurons using different fluorophores. The mitochondria in ChAT-Gal4 ${ }^{+}$neurons display robust motility and invariably good fluorescent signal in both the L1 and L3 wing veins. ChAT-Gal 4 is therefore an excellent alternative to pan-neuronal and more restricted drivers currently used in this system for live imaging of axonal transport $[35,52-55]$. We do not recommend the use of ubiquitous drivers as the fluorescent signal from non-neuronal cells in the wing would mask the signal from the axons of the marginal nerve.

8. We found that fusing KillerRed and SuperNova to one MTS results in diffuse cytoplasmic distribution of the proteins in Drosophila S2R+ cells, while using two MTS is sufficient for efficient mitochondrial targeting.

9. Make sure that the selected fly does not carry markers that affect wings morphology (e.g., CyO). Carefully check the wings at the stereo microscope. If damaged, discard the fly.

10. Wings not being flat on the coverslip will decrease the precision of the photostimulation and the clarity of the signal. The wing arch region (which is closer to the body) might not be flat on the bottom coverslip as a result of head and the thorax of the fly becoming unstuck from the coverslip, for instance if the head of the animal is not well attached on the double-sided tape. In this case, the application of little oil could be used to further restrain the animal. We found that finer round tip paintbrushes (for example, n.1) are particularly suitable to carefully position the fly.

11. It is possible to image mitochondrial transport in the wing for up to 30 minutes [35]. However, when imaging for longer periods of time, we recommend using a slower frame rate (e.g. 0.5 or $0.33 \mathrm{fps}$ ) to 
reduce phototoxicity. On the other hand, if the study focuses on the analysis of instantaneous velocities, recording at higher frame rates for a shorter period is more appropriate.

12. For good quality SRRF movies, a strong fluorescent signal and acquisition at high fps are essential. Therefore, we recommend against using dim fluorophores.

13. To obtain a single SRRF image, a series of frames needs to be acquired in a short time interval (e.g. 1 s) and exposure times between $10 \mathrm{~ms}$ and $100 \mathrm{~ms}$ are recommended [37].

14. The effective elapsed time between time series is defined by the combination of both the time needed to acquire 1 loop of 33 consecutive frames ( 1 second, with our settings) and the interval between each loop ( 2 seconds in our case). Our effective acquisition time is therefore of $0.33 \mathrm{fps}$. In an imaging period of $90 \mathrm{~s}$, this equals to 30 acquisition loops for a total of 990 frames. These settings allow to follow mitochondria reliably without significant photobleaching.

15. The settings were chosen by adjustment and comparison of several images. Ring Radius, used to calculate the intensity gradients of nearby subpixels, was chosen by iterative trials of different radii, until an optimal response of the analysis was observed, typically judged by the best resolution improvement and minimal patterning. Radiality Magnification, which determines the number of subpixels each pixel is split into, was not increased to more than 2 as improvements were not evident at greater values.

16. The size of the stimulation ROI, the scan speed and the laser intensity can influence the density of photons delivered per unit area. For each experiment, the stimulation ROI size, the number of loops, scan speed and laser intensity should be empirically optimised and then the settings kept constant throughout the experiment. In our experience, keeping the scan speed and laser intensity constant while adjusting the number of iterations, gives the most reliable results.

17. At constant laser power, high scanning speed and high number of loops result in more precise bleaching than a low scanning speed and low number of loops. For experimental design, it is useful to consider the time it takes to switch between optical configurations or stimulation loops, which may vary considerably depending on the microscope setup.

18. Our stimulation protocol is sufficient to trigger a rapid change in the redox state of the mitochondria. However, this recovers almost to pre-stimulation levels within 5 min (Figure 3C-D). If desired, longer and more frequent stimulation loops can be used which may result in sustained oxidation of the irradiated area. In this case, however, it is important to carefully control for potential light-induced mitochondrial oxidation.

19. The size of the ROI can be chosen depending on the specific experimental aim. To bleach moving mitochondria, the bigger the stimulation ROI the higher the probability that motile mitochondria will cross it at any given time. In the wing neurons, average mitochondria speed is approximately 0.2 $\mu \mathrm{m} / \mathrm{s}$ and $0.4 \mu \mathrm{m} / \mathrm{s}$ for anterograde and retrograde transport, respectively [47]. With a stimulation step of 10-15 s, the displacement of irradiated motile mitochondria could be of up to approximately 2-6 $\mu$ We found that using a 15-25- $\mu \mathrm{m}$-long stimulation ROI is sufficient for reliable bleaching of mito::KR in motile mitochondria. 
20. Ratiometric images were generated with the "RatioView" modality of NIS-element software. Alternatively, the Image Calculator command in Fiji can also be used.

21. Some areas immediately outside the bleached ROI are likely to be reached by the scattering of the excitation light and may respond to the stimulation protocol. This can be ascertained empirically and the extent of the irradiated sample outside the boundaries of the ROI minimised. When quantifying control regions (i.e., areas that have not been stimulated), it is therefore preferable to choose an area not adjacent to the irradiated ROI. Alternatively, using two-photon microscopy to perform the photostimulation experiments is likely to reduce the scattering of light in the tissue.

22. The wing nerve does not always appear in focus in the whole field of view when live flies are mounted. To reliably analyse mitochondria motility, select an area displaying minimal drifting where the nerve is flat on the coverslip.

\section{Declarations}

\section{Acknowledgements}

This work was funded through a NC3Rs David Sainsbury Fellowship and SKT grants (NC/N001753/2 and NC/T001224/1), a van Geest Ph.D. Studentship and a van Geest Fellowship in Dementia and Neurodegeneration from King's College London, a Royal Society Research Grant (RGS\R2\180450) and an Academy of Medical Sciences Springboard Award (SBF004\1088) to AV.

We would like to thank the Wohl Cellular Imaging Centre at King's College London for help with light microscopy and the Fly Facility of the Department of Genetics, University of Cambridge for help with Drosophila embryo injections.

This manuscript will be included in the forthcoming volume of Methods in Molecular Biology on 'Axonal transport'.

\section{Competing interests}

The authors declare no competing interests.

\section{References}

1. Sleigh JN, Rossor AM, Fellows AD, et al (2019) Axonal transport and neurological disease. Nat. Rev. Neurol. 15:691-703. https://doi.org/10.1038/s41582-019-0257-2

2. Badal K, Zhao Y, Miller K, Puthanveettil S (2021) Live imaging and quantitative analysis of organelle transport in sensory neurons of Aplysia Californica. Methods Mol Biol This volume.

3. Hees TJ, Harbauer AB (2021) Live-cell imaging of RNA transport in axons of cultured primary neurons. Methods Mol Biol This volume. 
4. Tierney W, Vicino I, Sun S, et al (2021) Methods and Applications of Campenot Trichamber Neuronal Cultures for the Study of Neuroinvasive Viruses. Methods Mol Biol This volume.

5. Ganguly A, Roy S (2021) Imaging diversity in slow axonal transport. Methods Mol Biol This volume.

6. Turchetto S, Le Bail R, Broix L, Nguyen L (2021) Molecular analysis of axonal transport dynamics upon modulation of microtubule acetylation. Methods Mol Biol This volume.

7. Shekari A, Fahnestock M (2021) Retrograde Axonal Transport of Neurotrophins in Basal Forebrain Cholinergic Neurons. Methods Mol Biol This volume.

8. Ionescu A, Perlson E (2021) Microfluidic neuromuscular co-culture system for tracking cell-to-cell transfer and axonal transport of labeled proteins. Methods Mol Biol This volume.

9. Montgomery A, Garbouchian A, Bentley M (2021) Visualizing vesicle-bound kinesins in cultured hippocampal neurons. Methods Mol Biol This volume.

10. Emily MF, Agrawal L, Barzaghi P, et al (2021) Use of microfluidics chambers to image axonal transport in adult sensory neurons. Methods Mol Biol This volume.

11. Mehta AR, Chandran S, Selvaraj BT (2021) Assessment of mitochondrial trafficking as a surrogate of fast axonal transport in human induced pluripotent stem cell derived spinal motor neurons. Methods Mol Biol This volume.

12. Voelzmann A, Sanchez-Soriano N (2021) Drosophila primary neuronal cultures as a useful cellular model to study and image axonal transport. Methods Mol Biol This volume.

13. Annuario EA, Ng KY-W (2021) High-resolution live imaging of mitochondria and mitochondrial nucleoids in differentiated SH-SY5Y cells. Methods Mol Biol This volume.

14. Smit-rigter L, Rajendran R, Silva CAP, et al (2016) Mitochondrial Dynamics in Visual Cortex Are Limited In Vivo and Not Affected by Axonal Structural Report Mitochondrial Dynamics in Visual Cortex Are Limited In Vivo and Not Affected by Axonal Structural Plasticity. Curr Biol 26:2609-2616. https://doi.org/10.1016/j.cub.2016.07.033

15. Turchetto S, Broix L, Nguyen L (2020) Ex Vivo Recording of Axonal Transport Dynamics on Postnatal Organotypic Cortical Slices. STAR Protoc 1:100131. https://doi.org/10.1016/j.xpro.2020.100131

16. Lin JQ, Cioni J-M (2021) Live imaging of RNA transport and translation in Xenopus retinal axons. Methods Mol Biol This volume.

17. Medioni C, Vijayakumar J, Ephrussi A, Besse F (2021) High-resolution live-imaging of axonal RNP granules in Drosophila pupal brain explants. Methods Mol Biol This volume.

18. Gould SA, Adalbert R, Milde S, Coleman M (2021) Imaging axonal transport in ex vivo central and peripheral nerves. Methods Mol Biol This volume.

19. Bateman JM (2021) Mitochondrial DNA transport in Drosophila neurons. Methods Mol Biol This volume.

20. Saxton WM, Lim A, Djagaeva I (2021) Dissection and direct imaging of axonal transport in drosophila segmental nerves. Methods Mol Biol This volume. 
21. Sleigh JN, Vagnoni A, Twelvetrees AE, Schiavo G (2017) Methodological advances in imaging intravital axonal transport. F1000Research 6:200. https://doi.org/10.12688/f1000research.10433.1

22. Huang $R, X u Y$, Chen $M$, et al (2021) Visualizing the intracellular trafficking in zebrafish Mauthner cells. Methods Mol Biol This volume.

23. Atkins $M$, Hazan J, Fassier C (2021) In vivo live imaging of axonal transport in developing zebrafish axons. Methods Mol Biol This volume.

24. Barmaver SN, Shanmugam MM, Wagner OI (2021) Methods to quantify and relate axonal transport defects to changes in C. ele-gans behavior. Methods Mol Biol This volume.

25. Knabbe J, Protzmann J, Kuner T (2021) In vivo imaging of axonal organelle transport in the mouse brain. Methods Mol Biol This volume.

26. Anazawa Y, Niwa S (2021) Analyzing the impact of gene mutations on axonal transport in Caenorhabditis elegans. Methods Mol Biol This volume.

27. Robinson W, Godenschwege TA (2021) Live imaging of axonal transport in the adult drosophila central nervous system. Methods Mol Biol This volume.

28. Nadiminti SSP, Koushika SP (2021) Imaging intracellular trafficking in neurons of C. elegans. Methods Mol Biol This volume.

29. Bearer E, Zhang X, Jacobs R (2021) Studying Axonal Transport in the Brain by Manganese-Enhanced Magnetic Resonance Imaging (MEMRI). Methods Mol Biol This volume.

30. Mattedi F, Vagnoni A (2019) Temporal Control of Axonal Transport: The Extreme Case of Organismal Ageing. Front Cell Neurosci 13:393. https://doi.org/10.3389/fncel.2019.00393

31. Knabbe J, Nassal JP, Verhage M, Kuner T (2018) Secretory vesicle trafficking in awake and anaesthetized mice: differential speeds in axons versus synapses. J Physiol 596:3759-3773. https://doi.org/10.1113/JP276022

32. Sleigh JN, Tosolini AP, Schiavo G (2020) In vivo imaging of anterograde and retrograde axonal transport in rodent peripheral nerves. Methods Mol Biol. 2143: 271-292. doi: 10.1007/978-1-07160585-1_20.

33. Halabi EA, Pinotsi D, Rivera-Fuentes P (2019) Photoregulated fluxional fluorophores for live-cell super-resolution microscopy with no apparent photobleaching. Nat Commun 10:1232. https://doi.org/10.1038/s41467-019-09217-7

34. Wang T, Li W, Martin S, et al (2020) Radial contractility of actomyosin rings facilitates axonal trafficking and structural stability. J Cell Biol 219:e201902001. https://doi.org/10.1083/jcb.201902001

35. Vagnoni A, Bullock SL (2016) A simple method for imaging axonal transport in aging neurons using the adult Drosophila wing. Nat Protoc 11:1711-1723. https://doi.org/10.1038/nprot.2016.112

36. Gustafsson N, Culley S, Ashdown G, et al (2016) Fast live-cell conventional fluorophore nanoscopy with ImageJ through super-resolution radial fluctuations. Nat Commun 7:1-9. https://doi.org/10.1038/ncomms12471 
37. Culley S, Tosheva KL, Matos Pereira P, Henriques R (2018) SRRF: Universal live-cell super-resolution microscopy. Int J Biochem Cell Biol 101:74-79. https://doi.org/10.1016/j.biocel.2018.05.014

38. Laine RF, Tosheva KL, Gustafsson N, et al (2019) NanoJ: A high-performance open-source superresolution microscopy toolbox. J Phys D Appl Phys 52:163001. https://doi.org/10.1088/13616463/ab0261.

39. Brand AH, Perrimon N (1993) Targeted gene expression as a means of altering cell fates and generating dominant phenotypes. Development 118:401-415.

40. Bulina ME, Chudakov DM, Britanova O V., et al (2006) A genetically encoded photosensitizer. Nat Biotechnol 24:95-99. https://doi.org/10.1038/nbt1175

41. Takemoto K, Matsuda T, Sakai N, et al (2013) SuperNova, a monomeric photosensitizing fluorescent protein for chromophore-assisted light inactivation. Sci Rep 3:2629.

https://doi.org/10.1038/srep02629

42. Albrecht SC, Barata AG, Grosshans J, et al (2011) In vivo mapping of hydrogen peroxide and oxidized glutathione reveals chemical and regional specificity of redox homeostasis. Cell Metab 14:819-829. https://doi.org/10.1016/j.cmet.2011.10.010

43. Grimm A, Cummins N, Götz J (2018) Local Oxidative Damage in the Soma and Dendrites Quarantines Neuronal Mitochondria at the Site of Insult. iScience 6:114-127. https://doi.org/10.1016/j.isci.2018.07.015

44. Onukwufor JO, Trewin AJ, Baran TM, et al (2020) Quantification of reactive oxygen species production by the red fluorescent proteins KillerRed, SuperNova and mCherry. Free Radic Biol Med 147:1-7. https://doi.org/10.1016/j.freeradbiomed.2019.12.008

45. Trewin AJ, Bahr LL, Almast A, et al (2019) Mitochondrial Reactive Oxygen Species Generated at the Complex-II Matrix or Intermembrane Space Microdomain Have Distinct Effects on Redox Signaling and Stress Sensitivity in Caenorhabditis elegans. Antioxid Redox Signal 31:594-607. https://doi.org/10.1089/ars.2018.7681

46. Gorbachev DA, Staroverov DB, Lukyanov KA, Sarkisyan KS (2020) Genetically Encoded Red Photosensitizers with Enhanced Phototoxicity. Int J Mol Sci 21:8800.

https://doi.org/10.3390/ijms21228800

47. Vagnoni A, Hoffmann PC, Bullock SL (2016) Reducing Lissencephaly-1 levels augments mitochondrial transport and has a protective effect in adult Drosophila neurons. J Cell Sci 129:178190. https://doi.org/10.1242/jcs.179184

48. Mangeol P, Prevo B, Peterman EJG (2016) KymographClear and KymographDirect: Two tools for the automated quantitative analysis of molecular and cellular dynamics using kymographs. Mol Biol Cell 27:1948-1957. https://doi.org/10.1091/mbc.E15-06-0404

49. Mórotz GM, Glennon EB, Greig J, et al (2019) Kinesin light chain-1 serine-460 phosphorylation is altered in Alzheimer's disease and regulates axonal transport and processing of the amyloid precursor protein. Acta Neuropathol Commun 7:200. https://doi.org/10.1186/s40478-019-0857-5 
50. Neumann S, Chassefeyre R, Campbell GE, Encalada SE (2017) KymoAnalyzer: a software tool for the quantitative analysis of intracellular transport in neurons. Traffic 18:71-88.

https://doi.org/10.1111/tra.12456

51. Jakobs MA, Dimitracopoulos A, Franze K (2019) Kymobutler, a deep learning software for automated kymograph analysis. Elife 8:e42288. https://doi.org/10.7554/eLife.42288

52. Vagnoni A, Bullock SL (2018) A cAMP/PKA/Kinesin-1 Axis Promotes the Axonal Transport of Mitochondria in Aging Drosophila Neurons. Curr Biol 28:1265-1272.e4. https://doi.org/10.1016/j.cub.2018.02.048

53. Neukomm LJ, Burdett TC, Gonzalez M a, et al (2014) Rapid in vivo forward genetic approach for identifying axon death genes in Drosophila. Proc Natl Acad Sci U S A 111:9965-9970. https://doi.org/10.1073/pnas.1406230111

54. Smith GA, Lin T-H, Sheehan AE, et al (2019) Glutathione S-Transferase Regulates Mitochondrial Populations in Axons through Increased Glutathione Oxidation. Neuron. 103:52-65.e6. https://doi.org/10.1016/j.neuron.2019.04.017

55. Fang Y, Soares L, Bonini NM (2013) Design and implementation of in vivo imaging of neural injury responses in the adult Drosophila wing. Nat Protoc 8:810-819. https://doi.org/10.1038/nprot.2013.042

56. Kaasik A, Safiulina D, Zharkovsky A, Veksler V (2007) Regulation of mitochondrial matrix volume. Am J Physiol Physiol 292:C157-C163. https://doi.org/10.1152/ajpcell.00272.2006

57. Long Q, Zhao D, Fan W, et al (2015) Article Modeling of Mitochondrial Donut Formation. Biophysj 109:892-899. https://doi.org/10.1016/j.bpj.2015.07.039

58. Verstreken P, Ohyama T, Haueter C, et al (2009) Tweek, an Evolutionarily Conserved Protein, Is Required for Synaptic Vesicle Recycling. Neuron 63:203-215. https://doi.org/10.1016/j.neuron.2009.06.017

59. Salvaterra PM, Kitamoto T (2001) Drosophila cholinergic neurons and processes visualized with Gal4/UAS-GFP. Gene Expr Patterns 1:73-82. https://doi.org/10.1016/S1567-133X(01)00011-4

60. Pilling AD, Horiuchi D, Lively CM, Saxton WM (2006) Kinesin-1 and Dynein are the primary motors for fast transport of mitochondria in Drosophila motor axons. Mol Biol Cell 17:2057-2068. https://doi.org/10.1091/mbc.E05-06-0526

61. Chowdhary S, Tomer D, Dubal D, et al (2017) Analysis of mitochondrial organization and function in the Drosophila blastoderm embryo. Sci Rep 7:1-17. https://doi.org/10.1038/s41598-017-05679-1

\section{Table}

Table 1. Drosophila stocks used in this study 


\begin{tabular}{|c|c|c|}
\hline Drivers and reporters & Purpose & Source \\
\hline$n S y b-G a l 4$ & $\begin{array}{l}\text { To express Gal4 pan-neuronally } \\
\text { in the wing sensory neurons }\end{array}$ & BDSC (\#68222) [58] \\
\hline ChAT-Gal4 & $\begin{array}{l}\text { To express Gal4 in the } \\
\text { cholinergic neurons of the wing }\end{array}$ & BDSC (\#56500)[59] \\
\hline UAS-mito::KillerRed & $\begin{array}{l}\text { To mark mitochondria and trigger } \\
\text { light-dependent ROS production }\end{array}$ & This study. \\
\hline UAS-mito::SuperNova & $\begin{array}{l}\text { To mark mitochondria and trigger } \\
\text { light-dependent ROS production }\end{array}$ & This study \\
\hline $\begin{array}{l}\text { UAS-mito::roGFP2- } \\
\text { Grx1 }\end{array}$ & $\begin{array}{l}\text { To mark and measure the } \\
\text { oxidation state of the } \\
\text { mitochondria }\end{array}$ & BDSC (\#67664)[42] \\
\hline UAS-mito::GFP & To mark mitochondria & BDSC (\#8442) [60] \\
\hline UAS-mito::PA-GFP & $\begin{array}{l}\text { To mark mitochondria after } \\
\text { activation with the } 405 \mathrm{~nm} \text { laser } \\
\text { line }\end{array}$ & $\begin{array}{l}\text { R. Rikhy (Indian Institute of Science } \\
\text { Education and Research, Pune, India) } \\
\text { [61] }\end{array}$ \\
\hline
\end{tabular}

\section{Figures}


A

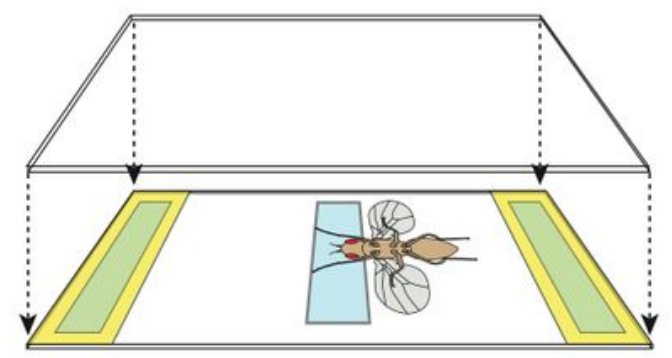

C
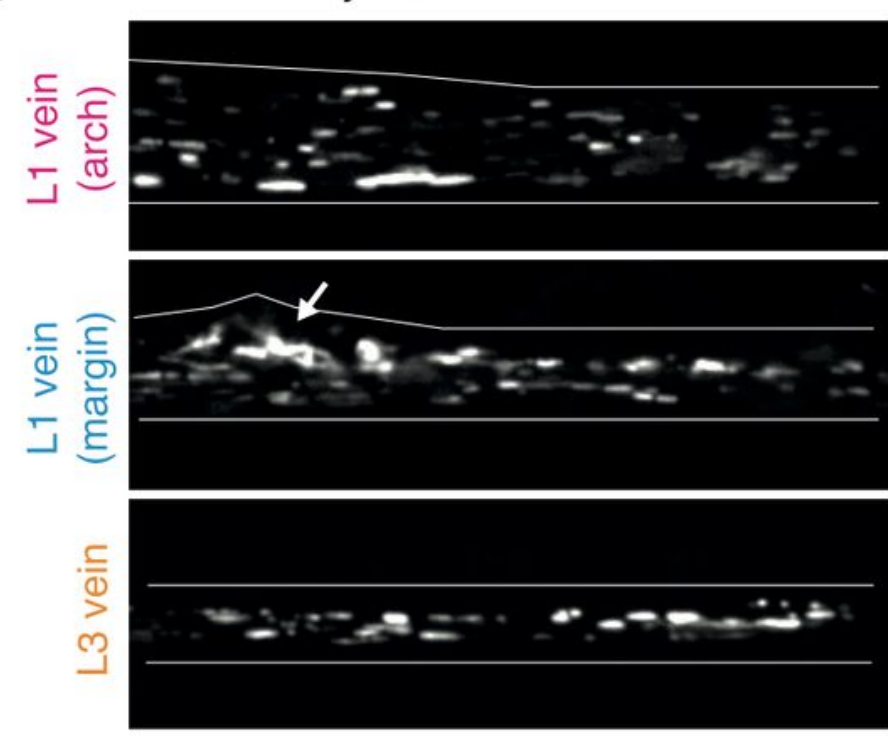

D

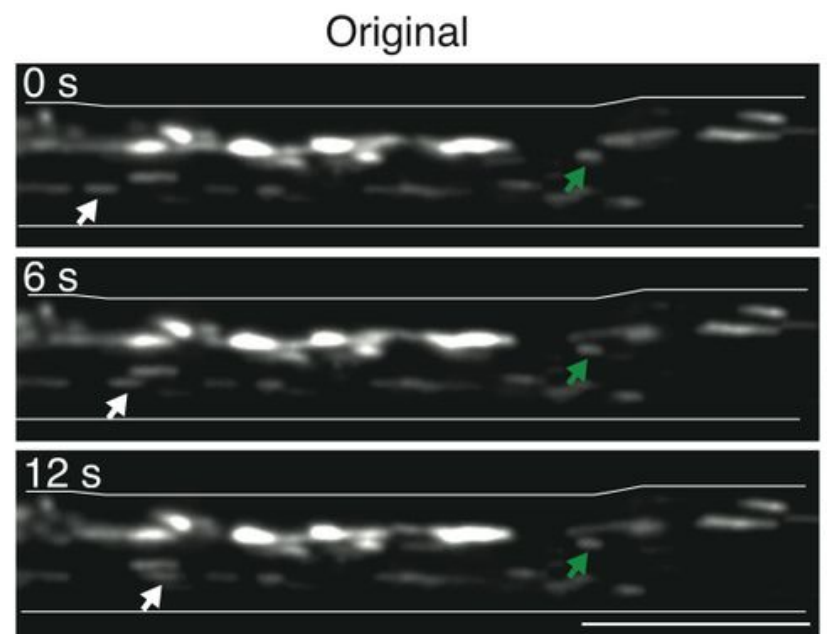

L1 vein (arch)

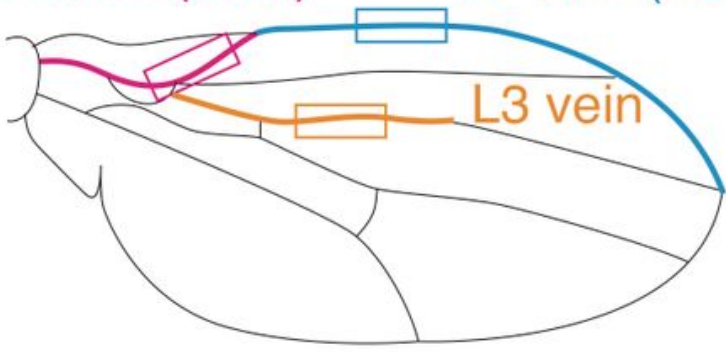

L1 vein (margin)

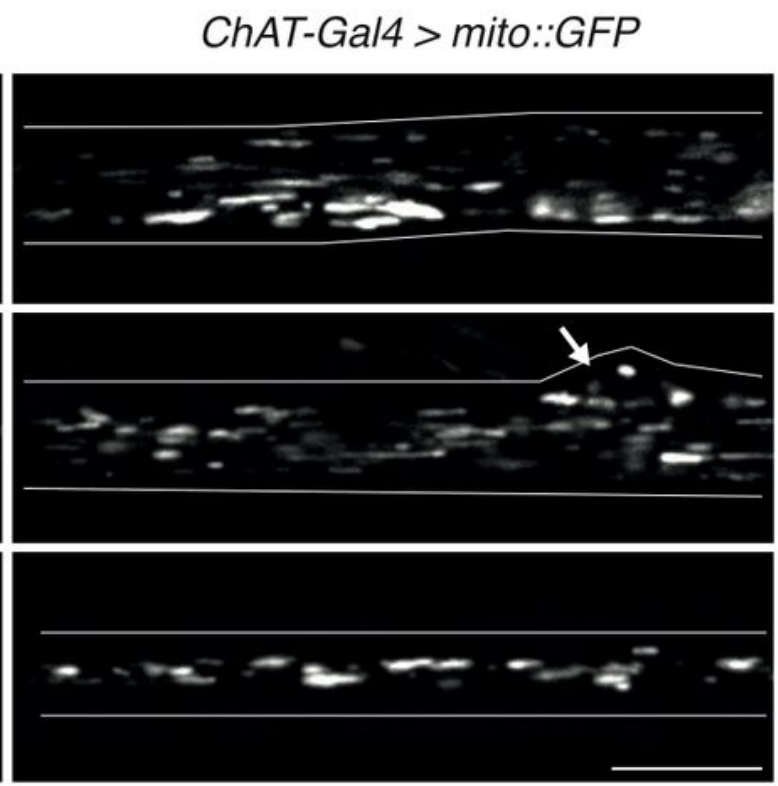

SRRF
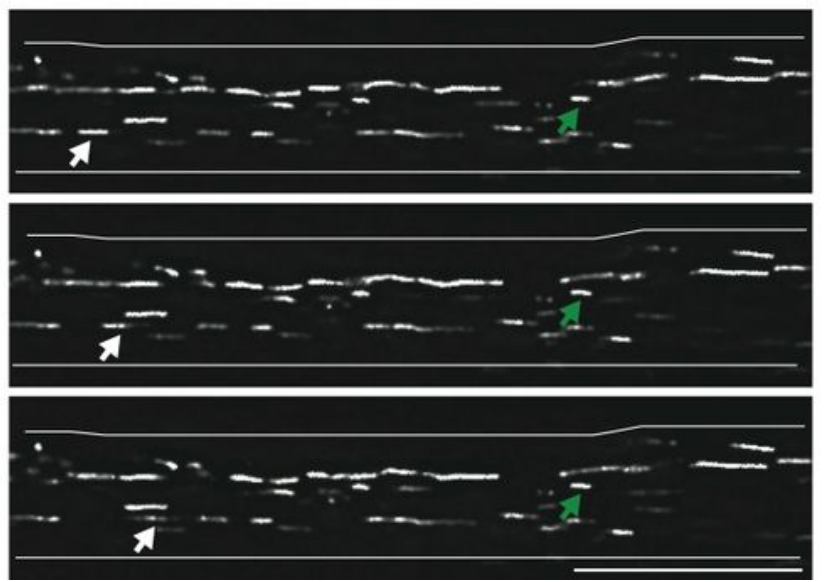

Figure 1

Imaging mitochondria in vivo in the neurons of the adult Drosophila wing. (A) Schematic of the chamber where the flies are mounted for imaging. Each of the short sides of a bottom coverslip are occupied by three layers of masking tape (yellow). A layer of double-sided tape (light blue) is attached onto the masking tape and in the centre of the coverslip. A second coverslip is used to seal the chamber. (B) Schematic of the wing with areas where live imaging is typically performed highlighted in colours. 
Rectangular selections represent the areas imaged in C. The arch of the L1 vein (magenta) and the L3 vein (orange) are areas of high axonal density with relatively low number of cell bodies and ideal for imaging studies of axonal transport. (C) Mitochondria marked by GFP expressed pan-neuronally (nSyb+ neurons, left panels) and in cholinergic neurons (ChAT+ neurons, right panels). Arrows indicate neuronal cells bodies. Scale bar: $10 \mu \mathrm{m}$. (D) Selected frames of a time-lapse movie (Movie 1) acquired from the wing arch before ('Original') and after ('SRRF') SRRF processing. The degree of resolution improvement was measured to range between 2.4 and 3.1 fold, with the average diameter of the mitochondria measured to be approximately $470 \mathrm{~nm}$ and $180 \mathrm{~nm}$ before and after SRRF analysis, respectively, which is in line with previous estimates [56, 57]. White arrow: moving mitochondrion. Green arrow: stationary mitochondrion. Genotype: ChAT-Gal4 > UAS-mito::GFP. In (C) and (D): white lines, outline of the wing nerve. Scale bar: $10 \mu \mathrm{m}$.

A

ChAT-Gal4 > mito::GFP, mito::KR
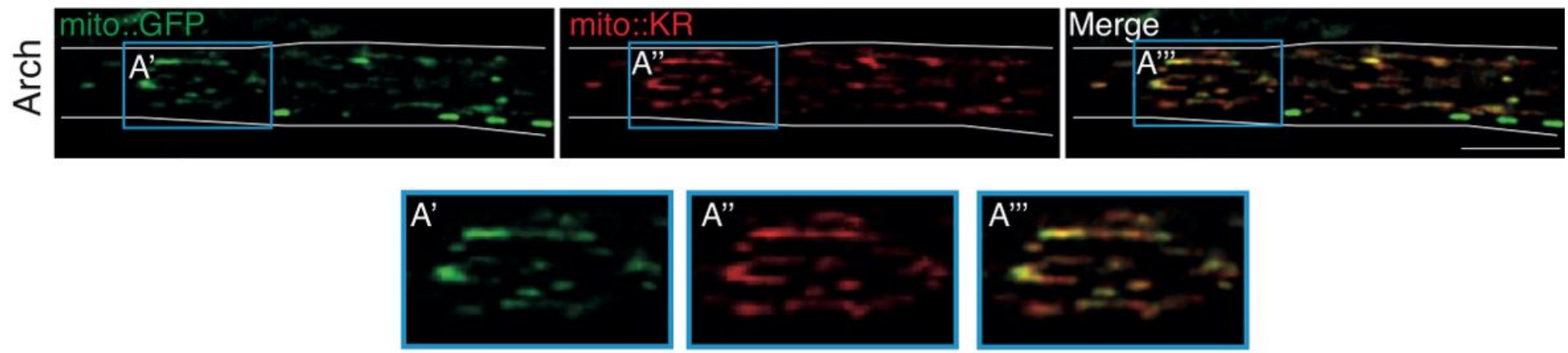

$\mathrm{B}$
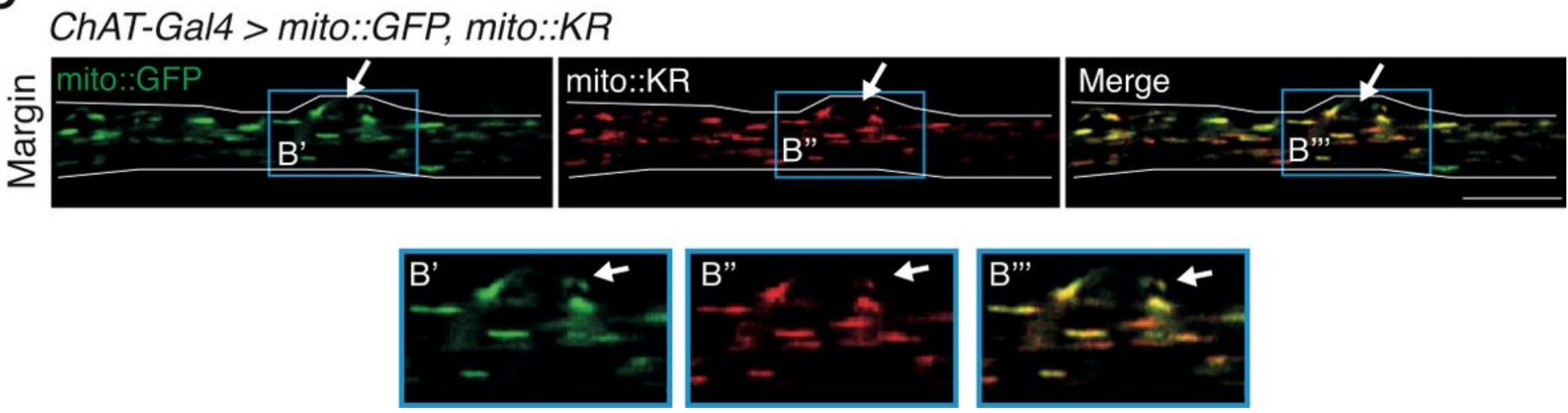

Figure 2

Mito::KR is localised to mitochondria in the wing nerve. Representative images of a $50-\mu \mathrm{m}$ long region from the arch (A) and margin (B) of the adult Drosophila wing. The vast majority of mitochondria are double stained with GFP and KillerRed. $A^{\prime}-A^{\prime \prime \prime}$ and $B^{\prime}-B^{\prime \prime}$ show magnified insets (blue rectangles) from $A$ and $B$, respectively. Arrows indicate neuronal cell bodies. In $(A)$ and $(B)$ : white lines, outline of the wing nerve. Scale bars: $10 \mu \mathrm{m}$. 
A

ChAT-Gal4 > mito::KR, mito::roGFP2-Grx1

$\sqrt{7}$

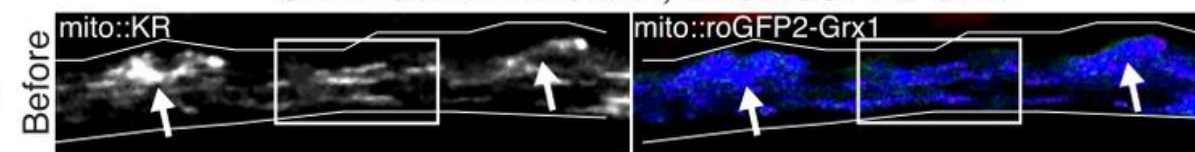

$561 \mathrm{~nm}$
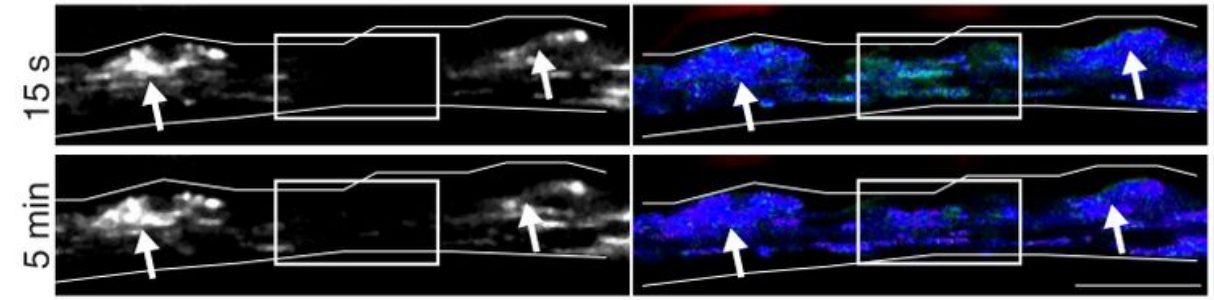

ChAT-Gal4 > mito::KR, mito::roGFP2-Grx1
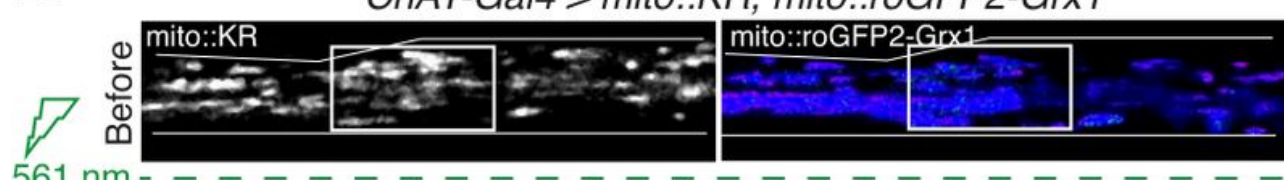

$561 \mathrm{~nm}$
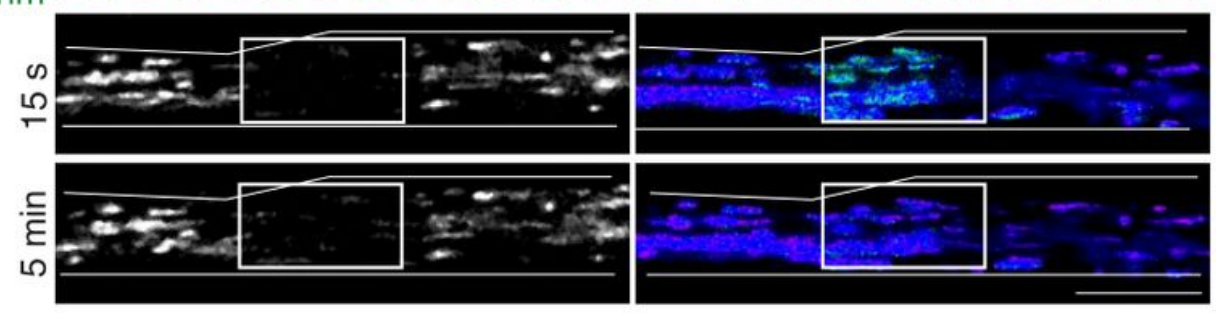

ChAT-Gal4 > mito::roGFP2-Grx1
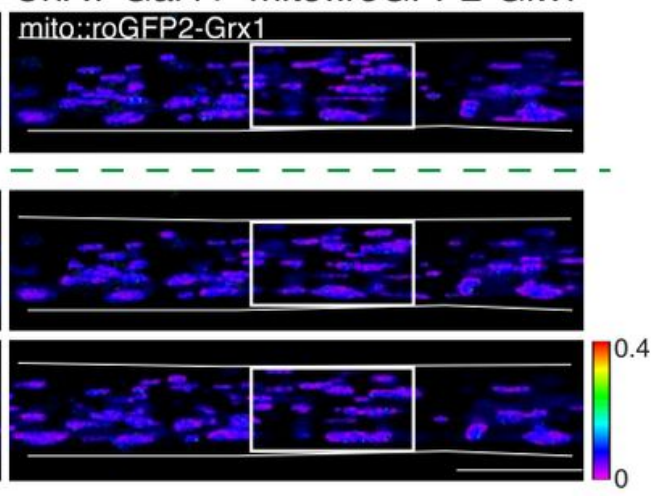

C

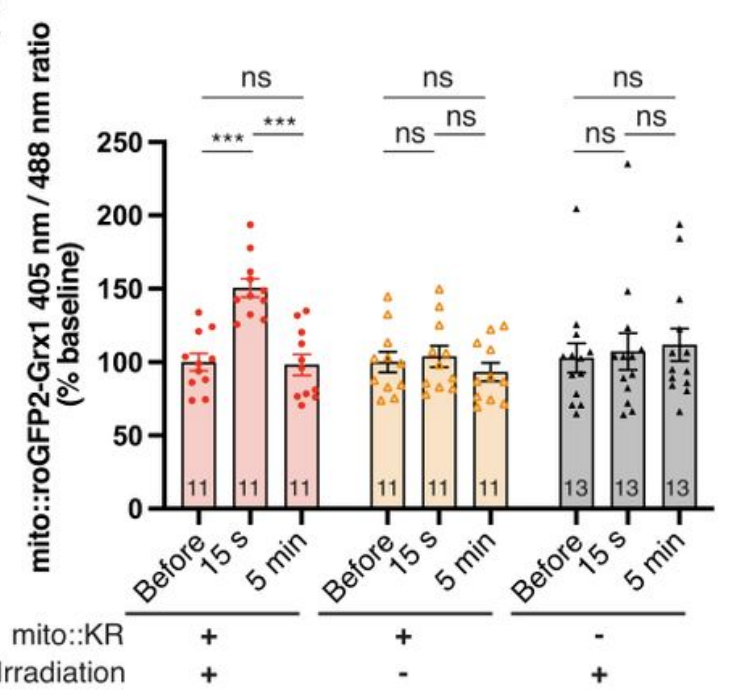

D

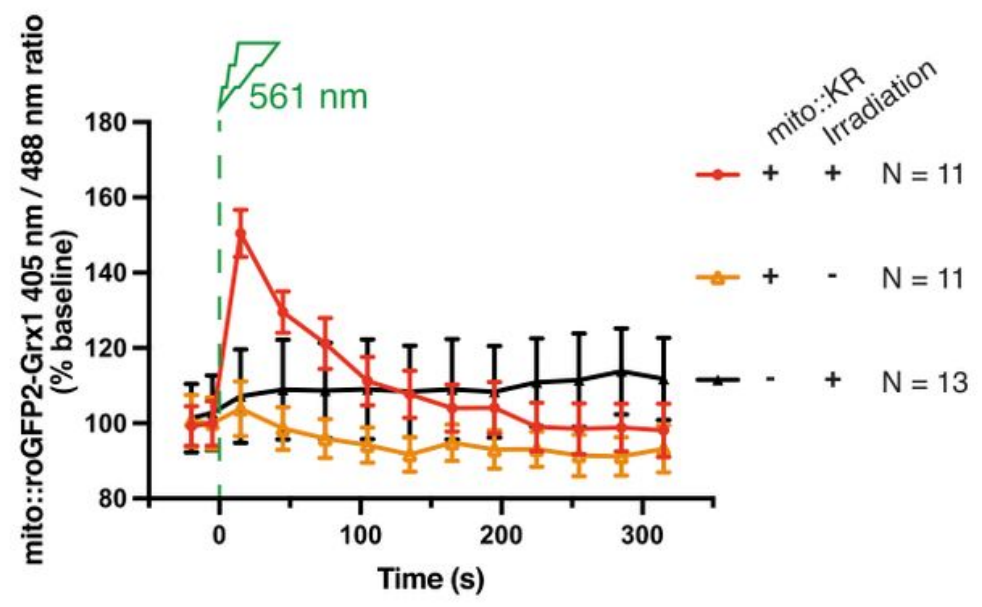

\section{Figure 3}

Photostimulation of mito::KR results in a local increase in mitochondrial ROS. (A, B) Left panels: mito::KR signal before and after 561-nm irradiation (dashed green line) shows efficient bleaching of mito::KR in the axons of the marginal wing nerve (A) and arch region (B). Middle panels: ratiometric images reveal a rapid local increase in mitochondrial ROS shortly after irradiation. Mitochondrial oxidation returns to prebleach levels within 5 minutes. Right panels: control neurons lacking mito::KR do not display an increase in mitochondrial ROS. In all panels, the bleached area is marked by white rectangles and the white lines mark the outline of the wing nerve. Arrows indicate neuronal cell bodies. Scale bars: $10 \mu \mathrm{m}$. (C) 
Quantification of the mitochondrial redox state in the wing arch before mito::KR bleaching, 15 seconds after bleaching and at the end of the experimental series, as shown in (B). Repeated Measures One-Way Anova with Tukey's post-hoc test. ns $=$ not significant $(p>0.05)$, $* \star * p<0.001$. (D) Quantification of the mitochondrial redox state in the wing arch for the entire experimental series. The 405/488 nm ratio was measured at two timepoints before bleaching (dashed green line) and at 30-s intervals after that for five minutes. In (C) and (D), 'baseline' is the mean 405/488 nm ratio prior to stimulation. Quantifications of non-irradiated areas ('- Irradiation') are performed on the same UAS-mito::KR flies that were subject to irradiation ('+ Irradiation'), as described in Section 3.9. Error bars are mean \pm s.e.m. N: number of wings, one ROI per wing was selected for bleaching.

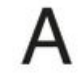

ChAT-Gal4 > mito::KR, mito::roGFP2-Grx1
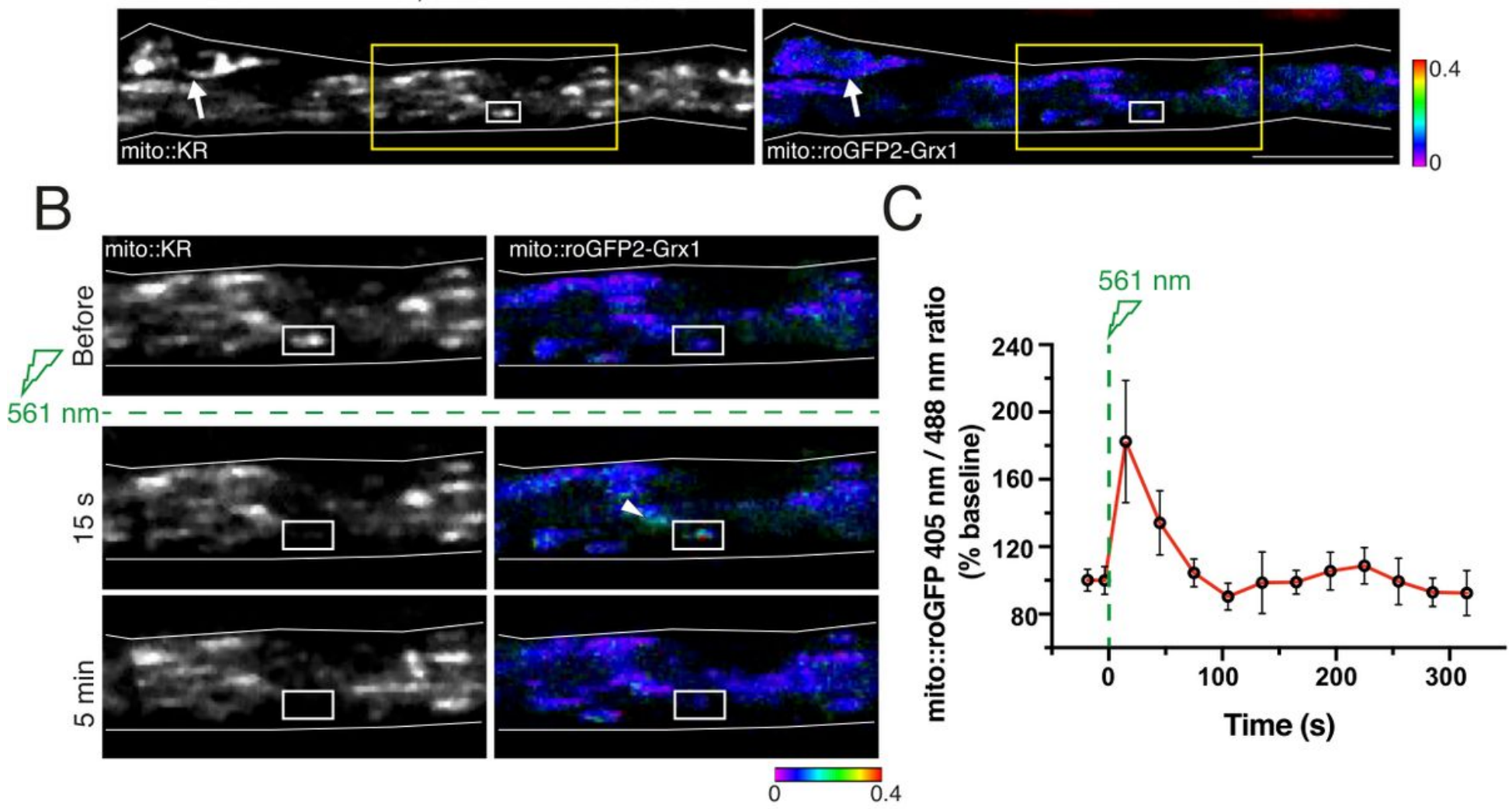

Figure 4

Restricted photostimulation of mito::KR results in ROS increase in single mitochondria. (A) Representative images of the neurons in the wing margin before stimulation. White box: stimulation ROI, yellow box: magnified insets in B. Arrows indicate neuronal cell bodies. Scale bar: $10 \mu \mathrm{m}$ (B) Left panels: mito::KR signal before and after 561-nm irradiation (dashed green line) shows precise and efficient bleaching of mito::KR in a single mitochondrion of the marginal wing. Right panels: ratiometric images reveal a rapid local increase in mitochondrial ROS shortly after irradiation. Mitochondrial oxidation returns to pre-bleach levels within 5 minutes. In all panels, the bleached area is marked by white rectangles. Despite the precise bleaching of the mito::KR signal, a change in the 405/488 nm ratio in the immediate surrounding of the bleached area was also observed 15 seconds post-bleaching (white arrowhead). This might be due to light scattering during the bleaching protocol that could activate mito::KR in close proximity of the 
stimulated ROI (see Note 21). In (A) and (B): white lines, outline of the wing nerve. (C) Quantification of the redox state in single mitochondria of the wing margin. The $405 / 488 \mathrm{~nm}$ ratio was measured at two timepoints before bleaching (dashed green line) and at 30-s intervals after that for five minutes. Scale bars are mean \pm s.e.m. $\mathrm{N}=3$ mitochondria.

\section{A ChAT-Gal4 > mito::GFP, mito::SN}
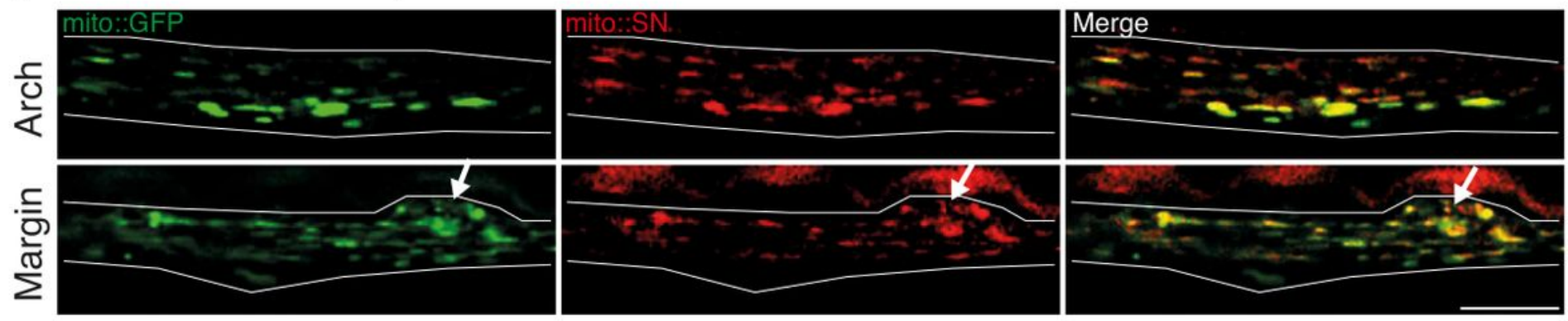

B ChAT-Gal4 > mito::SN, mito::roGFP2-Grx1
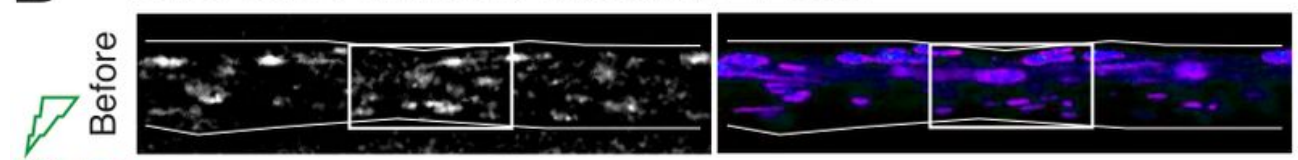

ChAT-Gal4 > mito::roGFP2-Grx1

$561 \mathrm{~nm}-$
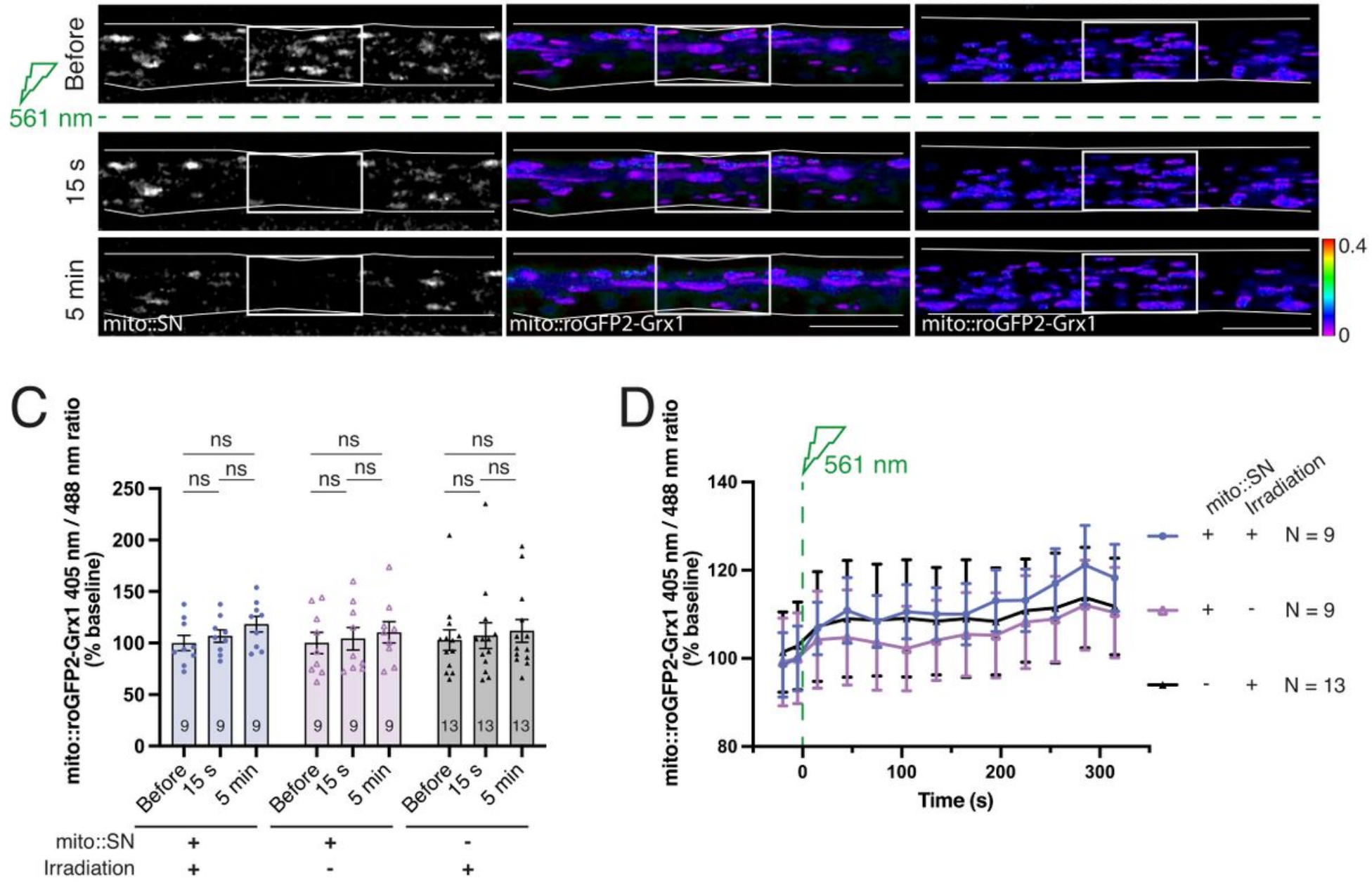

\section{Figure 5}

Mito::SN is localised to mitochondria in the wing nerve. (A) Representative images of a $50-\mu \mathrm{m}$ long region from the arch (top panels) and margin (bottom panels) of the adult Drosophila wing. The vast majority of mitochondria are double stained with GFP and SuperNova. Arrows indicate neuronal cell bodies. Scale bar: $10 \mu \mathrm{m}$. (B) Left panels: mito::SN signal before and after 561-nm irradiation (dashed green line) 
shows efficient bleaching of mito::SN in the axons of arch region. Middle panels: ratiometric images show no significant increase in mitochondrial ROS after irradiation. Right panels: control neurons lacking mito::SN do not display an increase in mitochondrial ROS. In all panels, the bleached area is marked by white rectangles and the white lines mark the outline of the wing nerve. Scale bars: $10 \mu \mathrm{m}$. (C) Quantification of the mitochondrial redox state in the wing arch before mito::SN bleaching, 15 seconds after bleaching and at the end of the experimental series, as shown in (B). (D) Quantification of the mitochondrial redox state in the wing arch for the entire experimental series. The 405/488 nm ratio was measured at two timepoints before bleaching (dashed green line) and at 30-s intervals after that for five minutes. Error bars are mean \pm s.e.m. N: number of wings, one ROI per wing was selected for bleaching. In (C) and (D), 'baseline' is the mean $405 / 488 \mathrm{~nm}$ ratio prior to stimulation. Quantifications of nonirradiated areas ('- Irradiation') are performed on the same UAS-mito::SN flies that were subject to irradiation ('+ Irradiation'), as described in Section 3.9. Repeated Measures One-Way Anova with Tukey's post-hoc test. Ns $=$ not significant $(p>0.05)$.

A

Pre irradiation

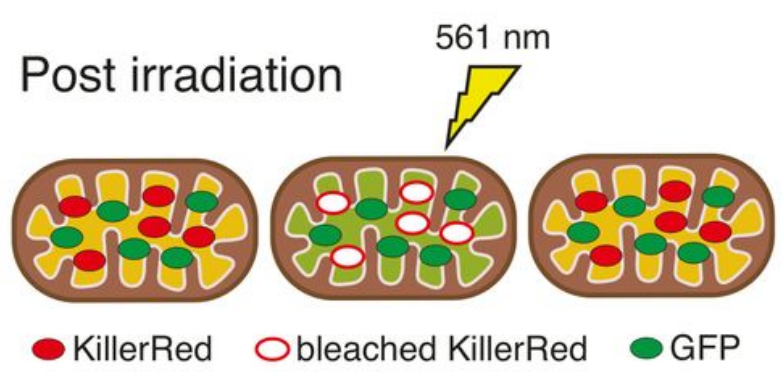

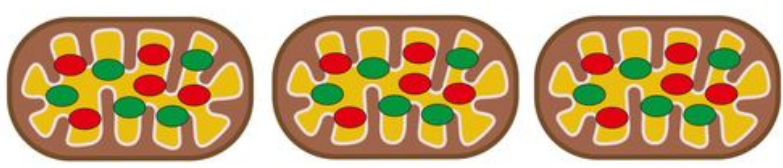

KillerRed GFP

\section{KillerRed Obleached KillerRed OGFP}

Pre irradiation

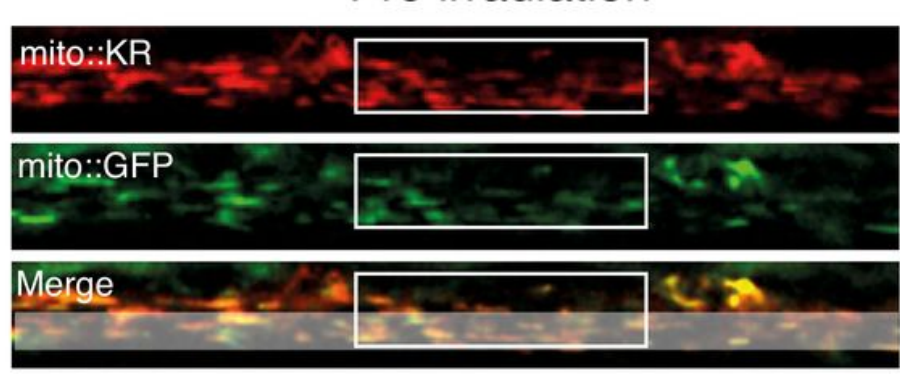

Post irradiation

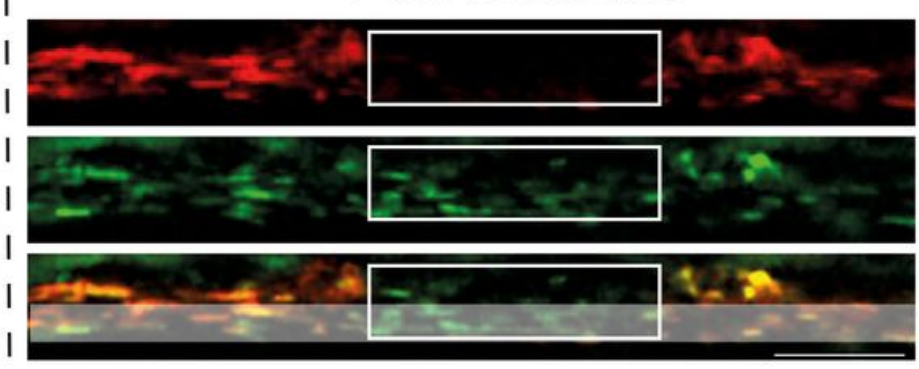

C

ChAT-Gal4 > mito::KR, mito::GFP

$561 \mathrm{~nm}$
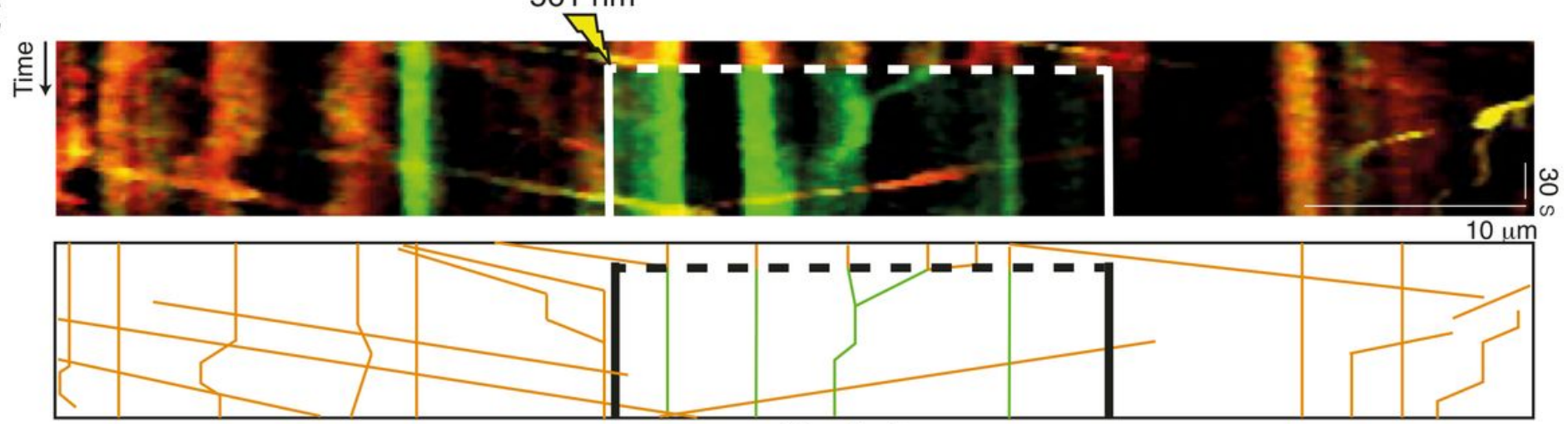

Bleached area 


\section{Figure 6}

Assessing mitochondrial motility after acute mitochondrial damage using mito::KR and mito::GFP. (A) The cartoon illustrates the experimental design. After bleaching KillerRed with the 561-nm laser, mitochondria undergo a yellow-to-green transition, indicated by the change in the colour of the mitochondrial matrix in the cartoon: the red KillerRed signal is quenched and only the green GFP fluorescence is retained in the mitochondria. Bleached (i.e., dysfunctional) mitochondria can therefore be followed by illumination with both 488-nm and 561-nm lasers. (B) Neurons in the margin of the wing nerve before and after irradiation (dashed black line). The white rectangles indicate the photostimulated ROI. The area shaded in the bottom panels was used to generate the kymograph in (C). Scale bar: $10 \mu \mathrm{m}$. (C) Kymograph indicating a representative portion of stationary and motile mitochondria before and after KillerRed bleaching. The white and black rectangles (top and bottom panels, respectively) delimit the bleached area, with the dashed line indicating the time of bleaching. Only mitochondria that intersect the dashed line were bleached. Bottom panel: orange-to-green tracks: bleached mitochondria, while in motion or stationary. Orange tracks: unbleached mitochondria. 


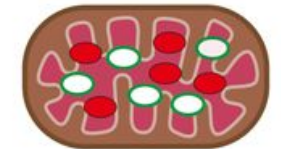

- KillerRed

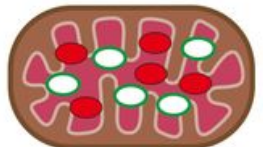

Onon-activated PA-GFP
Post irradiation

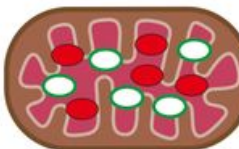

-KillerRed

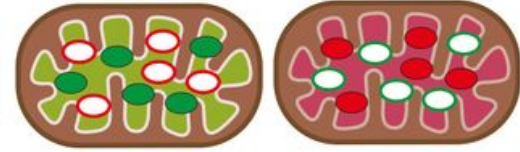

Onon-activated PA-GFP
O bleached KillerRed activated PA-GFP

B

ChAT-Gal4 > mito::KR, mito::PA-GFP

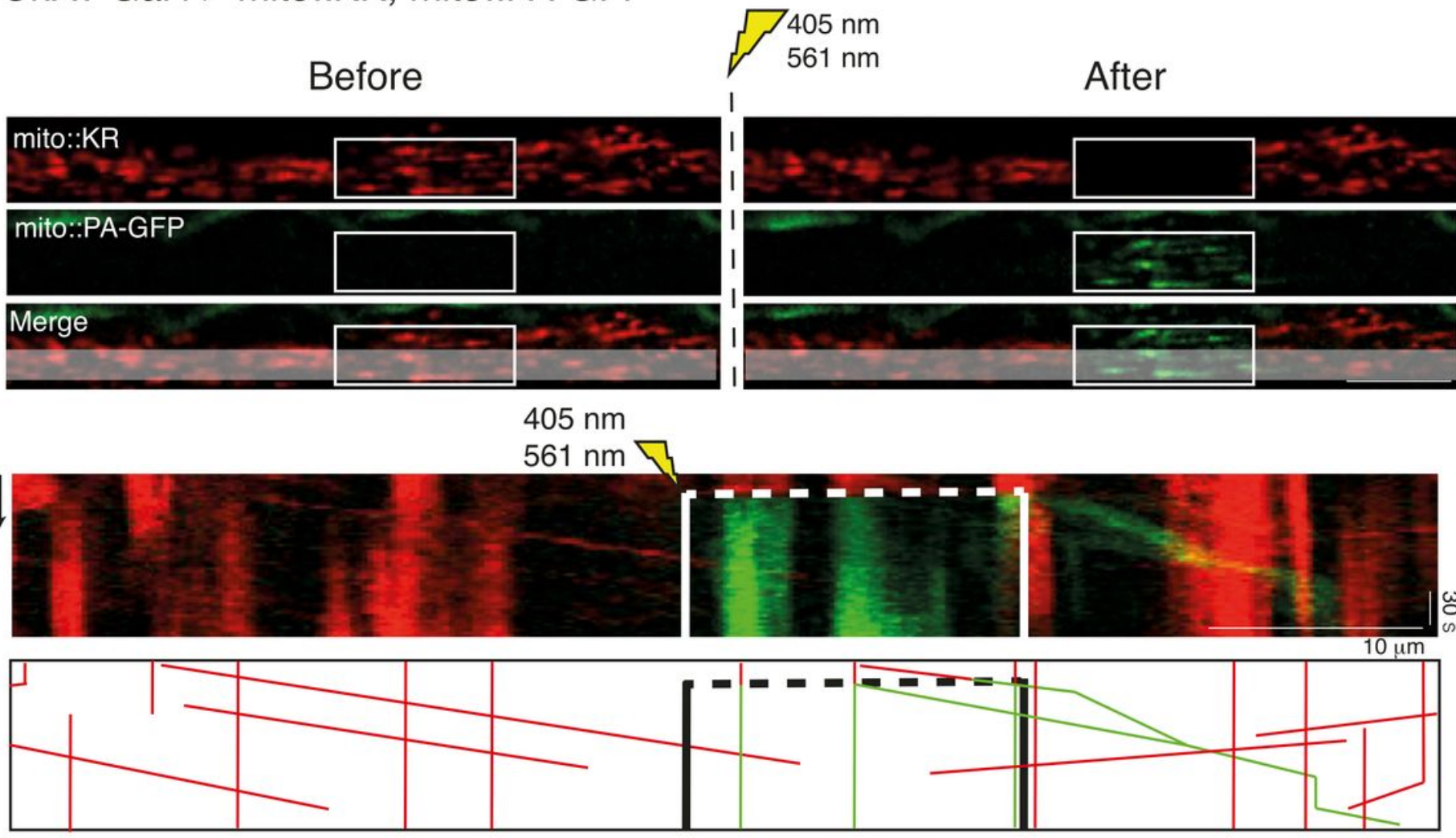

Bleached and

photoactivated area

\section{Figure 7}

Assessing mitochondrial motility after acute mitochondrial damage using mito::KR and mito::PA-GFP. (A) The cartoon illustrates the experimental design. After simultaneously bleaching KillerRed and photoactivating the GFP, mitochondria undergo a red-to-green transition, indicated by the change in the colour of the mitochondrial matrix in the cartoon: the red KillerRed signal is quenched while the green GFP fluorescence appears in the mitochondria. Bleached (i.e., dysfunctional) mitochondria can therefore be followed by illumination with the $488 \mathrm{~nm}$ laser only. (B) Neurons in the margin of the wing nerve before and after irradiation with the $405 \mathrm{~nm}$ and $561 \mathrm{~nm}$ lasers (dashed black line). The white rectangles indicate the stimulated ROI. The area shaded in the bottom panels was used to generate the kymograph in (C). Scale bar: $10 \mu \mathrm{m}$. (C) Kymograph indicating a representative portion of stationary and motile mitochondria before and after KillerRed bleaching and PA-GFP activation. The white and black rectangles (top and bottom panels, respectively) delimit the bleached area, with the dashed line indicating the time 
of bleaching. Only mitochondria that intersect the dashed line were bleached and photoactivated. Bottom panel: red-to-green tracks: bleached mitochondria, while in motion or stationary. Red tracks: unbleached mitochondria.

\section{Supplementary Files}

This is a list of supplementary files associated with this preprint. Click to download.

- Movie1.mov

- Movie2.mov

- Movie3.mov 